\title{
Linfohistiocitosis hemofagocítica secundaria y síndrome de liberación de citoquinas en COVID-19, ¿entidades iguales o diferentes?
}

\author{
Secondary hemophagocytic lymphohistiocytosis and cytokine release syndrome in \\ COVID-19, the same or different entities?
}

\author{
Alberto Alfaro-Murillo ${ }^{1,3}$ y Gustavo Lazo-Paéz ${ }^{2,3}$
}

'Medicina Interna e Inmunología Clínica, Caja Costarricense del Seguro Social. San José, Costa Rica.
${ }^{2}$ Pediatría e Inmunología Clínica, Servicio de Inmunología y Reumatología Pediátrica, Hospital Nacional de Niños "Dr. Carlos Sáenz-Herrera". San José, Costa Rica.
${ }^{3}$ Sistema de Estudios de Posgrado, Universidad de Costa Rica.

Los autores declaran no tener financiamiento externo.

Conflicto de intereses: ninguno.

Recibido: 17 de julio de 2020 / Aceptado: 5 de febrero de 2021

\section{Resumen}

Introducción: La liberación excesiva de citoquinas en COVID-19 grave se asemeja a la linfohistiocitosis hemofagocítica secundaria (sHLH). Objetivo: Comparar las características clínicas y de laboratorio entre sHLH y el síndrome de liberación de citoquinas (CRS) en COVID-19. Métodos: Se realizó una revisión de artículos en la base de datos PubMed, a través de las siguientes palabras clave " $H L H$ and COVID", "HScore in COVID". Se incluyeron las publicaciones disponibles hasta el 16 julio 2020. Resultados: Se elaboró un cuadro comparativo basado en los criterios diagnósticos del protocolo HLH 2004, HScore y características del CRS-COVID-19. Se utilizaron 18 variables para la comparación. Discusión: El CRS en COVID-19 grave presenta similitud con el CRS del sHLH; sin embargo, no se puede afirmar que se traten de la misma entidad. Los reportes de sHLH en COVID-19 son escasos. HScore es una herramienta que podría orientar el diagnóstico de HLH secundario a COVID-19 de una manera más práctica que los criterios HLH-2004; sin embargo, su aplicación en COVID-19 se encuentra limitada debido a la ausencia de características claves del estado hiperinflamatorio de COVID-19 que sí destacan en HLH. Conclusiones: El CRS-COVID-19 no es sinónimo de sHLH. Aunque esta última entidad podría o no estar presente en COVID-19 grave.

Palabras clave: citocinas; síndrome de activación macrofágica; linfohistiocitosis hemofagocítica; coronavirus; síndrome respiratorio agudo grave; virus del SARS.

\begin{abstract}
Background: Excessive release of cytokines in severe COVID-19 resembles secondary hemophagocytic lymphohistiocytosis (sHLH). Aim: To compare the clinical and laboratory characteristics between sHLH and cytokine release syndrome (CRS) in COVID-19. Methods: A review of articles in the PubMed database was performed, using the following keywords "HLH and COVID", "HScore in COVID". Articles available until July 16, 2020 were included. Results: A comparative table was prepared based on the diagnostic criteria of the HLH 2004 protocol, HScore and characteristics of the CRSCOVID-19. Eighteen variables are used for comparison. Discussion: The CRS in COVID-19 presented similarity with the CRS of sHLH; however, it cannot be stated that they are the same entity. Case reports of sHLH in COVID-19 are small. HScore is a tool that could guide the diagnosis of SHLH in the context of CRS-COVID-19, in a more practical way than the classic criteria described in HLH-2004; however, its application in COVID-19 is limited due to the absence of key features of the hyperinflammatory state of COVID-19 that are included in HLH. Conclusions: CRS-COVID-19 is not synonymous with sHLH. Although this last entity may or may not be present in the severe COVID-19.

Keywords: cytokines; macrophage activation syndrome; lymphohistiocytosis; hemophagocytic; coronavirus; severe acute respiratory syndrome; SARS virus.
\end{abstract}

Gustavo Lazo-Paéz

gustavo.lazo@gmail.com 


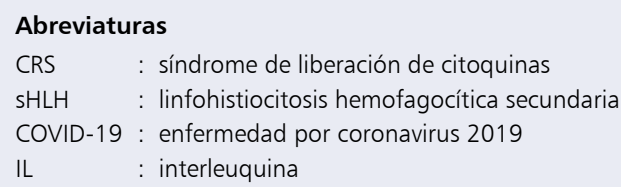

\section{Introducción}

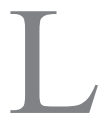
a pandemia 2020 por SARS-CoV-2 sacude actualmente la región de las Américas con una cantidad creciente de casos infectados y muertes. Se ha descrito que los casos graves por COVID-19 se caracterizan por una liberación excesiva de citoquinas acompañando al distrés respiratorio grave. Esto traduce un estado de disregulación inmunológica en los pacientes con neumonía grave por SARS-CoV-2 ${ }^{1}$. Si bien es cierto que la inflamación es parte indispensable en el proceso de defensa del hospedero contra distintos patógenos, en la defensa contra SARS-CoV-2 ocurre un desbalance inflamatorio, con consecuente hiperactivación inmune y daño tisular, no dilucidado por completo hasta el momento $^{2}$. Esta condición hiperinflamatoria es particularmente propagada por la liberación de péptidos secretados por distintos tipos de leucocitos, llamados citoquinas.

Este estado disregulatorio se denomina síndrome de liberación citoquínica o tormenta citoquínica (en inglés cytokine release syndrome - CRS). El CRS no es un término nuevo ni exclusivo de la enfermedad grave por SARS-CoV-2. Fue descrito por primera vez en 1993 en el contexto de enfermedad injerto versus hospedero en trasplante alógenico de células madre hematopoyéticas ${ }^{3}$; sin embargo, el CRS se ha descrito también en shock séptico de cualquier etiología infecciosa, terapia de CAR T cell (del inglés chimeric antigen receptor), shock anafiláctico ${ }^{4}$, entre otros. Para el diagnóstico de CRS se formularon los criterios de Lee y cols., en el contexto de CRS secundario a la infusión de CAR T cell (Cuadro 1) ; $^{5}$ sin embargo, son amplios en su definición, y en el caso de extrapolar estos criterios al CRS relacionado con COVID19, se excluyen parámetros importantes de laboratorio que se han descrito en el paciente con COVID-19 grave.

Por otra parte, de acuerdo con los distintos reportes publicados hasta el momento, esta liberación excesiva de citoquinas en COVID-19 grave se asemeja a una condición denominada linfohistiocitosis hemofagocitica secundaria $(\mathrm{sHLH})^{6}$. Esta es una condición potencialmente mortal que se caracteriza por una activación desenfrenada de los linfocitos T citotóxicos, las células NK y los macrófagos. Su causa puede ser primaria, debido a trastornos en la liberación y exocitosis de gránulos citotóxicos en el espacio denominado sinapsis inmunológica, a causa de trastornos genéticos, usualmente con patrón de herencia autosómico recesivo; o bien secun- daria, debido a etiologías infecciosas (virales, fúngicas, bacterianas y parasitarias), etiologías neoplásicas o bien enfermedades autoinmunes; para estas últimas se reserva de manera exclusiva el nombre de síndrome de activación macrofágica ${ }^{7,8}$. HLH se caracteriza al igual que el CRSCOVID-19 por una dramática elevación de citoquinas pro-inflamatorias a mencionar: IL-1 $\beta$, IL-2, IL-6, IL-12, IL-16, IL-18, FNT $\alpha$ e IFN $\gamma^{7}$ (Figura 1).

Para el diagnóstico de esta entidad, se han validado los criterios contenidos en el estudio de HLH primario pediátrico (HLH 1994), reformulados en el protocolo HLH-2004, utilizados hasta la fecha9. A pesar de ello, estos criterios fueron formulados para pacientes bajo 18 años de edad en los que se sospechara de HLH primario; además, los criterios diagnósticos contemplan las concentraciones del receptor soluble de IL-2 y la actividad de las células NK, situación que hace poco práctico el empleo de estos criterios para el ejercicio clínico, debido a que no se encuentra fácilmente este recurso en los laboratorios clínicos, además de que no fueron validados para población adulta9 ${ }^{9}$. En el 2014, se plantean los criterios de HScore, los cuales sí fueron validados para el sHLH en el adulto ${ }^{10}$.

El objetivo principal de esta revisión, es comparar las características clínicas, fisiopatológicas y de laboratorio entre sHLH (mediante los criterios diagnósticos descritos en el protocolo HLH 2004 y el HScore 2014) y el síndrome de liberación de citoquinas relacionado con COVID-19 (CRS-COVID-19), ambas condiciones potencialmente mortales. El propósito es brindar al médico clínico herramientas prácticas para el diagnóstico de estas entidades y así determinar si se tratan de la misma condición patológica.

\section{Métodos}

Se procedió a buscar publicaciones disponibles en la base de datos PubMed, en las que se hiciera mención a "HLH and COVID", "HScore in COVID". Se consideraron para el análisis las publicaciones disponibles al 16 julio de 2020. Se encontró un total de 24 publicaciones, estrictamente bajo la búsqueda de las palabras clave mencionadas. Se excluyeron del análisis aquellas publicaciones referentes a tratamientos específicos sugeridos para casos graves de COVID-19.

Para establecer comparaciones entre estos descriptores, se construyó un cuadro de referencia basado en el protocolo HLH 2004 y HScore. Éstos se comparan con el CRS propio de la infección grave por SARS-CoV-2 ${ }^{11-12}$.

Las comparaciones se clasificaron en parámetros clínicos, de laboratorio y otros parámetros varios. Se colocaron observaciones al lado de cada descriptor con el fin de aclarar lo mejor posible similitudes y diferencias entre el CRS-COVID-19, HLH 2004 y HScore. 
Cuadro 1. Criterios y clasificación por grados del síndrome de liberación de citoquinas (CRS)*

Grado 1 Los síntomas no son potencialmente mortales y requieren únicamente tratamiento sintomático. Incluye fiebre, náuseas, fatiga, malestar general

Grado 2 Los síntomas requieren y responden a una intervención limitada:

- Requerimiento de oxígeno $<40 \%$, $\leq 3$ L nasocánula o

- Hipotensión que responde a fluidos o dosis baja de 1 vasopresor o

- Grado 2 toxicidad renal o hepática

Grado 3 Los síntomas requieren y responden a una intervención agresiva:

- Requerimiento de oxígeno $\geq 40 \%$, > 3 L nasocánula o

- Hipotensión que requiere dosis altas o múltiples vasopresores o

- Toxicidad renal de grado 3 o transaminitis de grado 4

- Alteración de novo del estado de consciencia sin otra explicación**

-Nueva miocardiopatía sin anormalidad en el movimiento de la pared.

Grado 4 Síntomas que amenazan la vida:

- Ventilación mecánica asistida

- Toxicidad renal de grado 4 (excluyendo transaminitis)

Grado $5 \quad$ Muerte

**Alteración del estado mental de suficiente intensidad que requiera imágenes de cráneo y/o punción lumbar. *Modificado de ref 5.

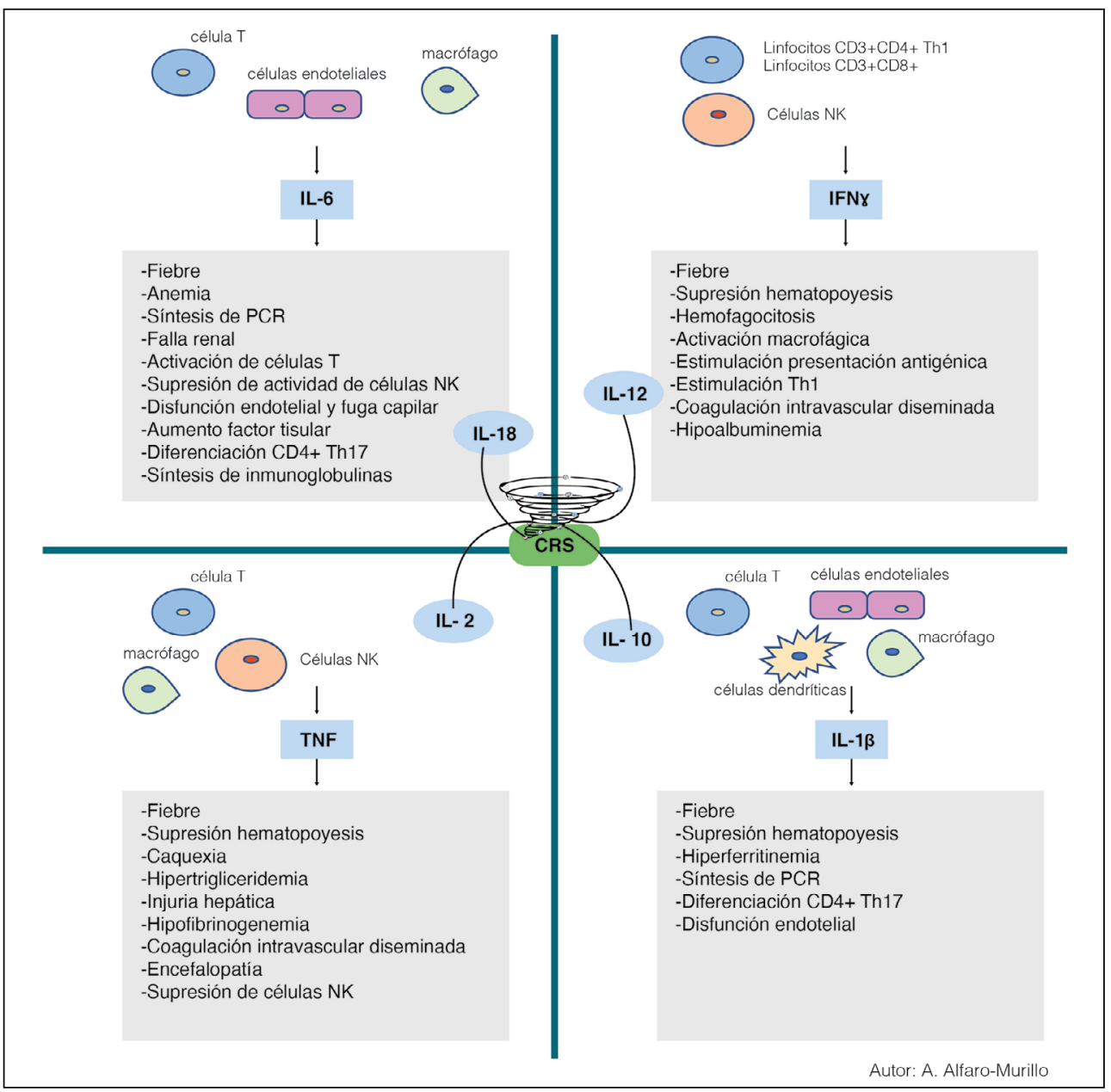

Figura 1. Representación del síndrome de tormenta de citoquinas en HLH y CRS-COVID-19. Se muestran las principales interleuquinas involucradas en la patogenia y sus principales funciones biológicas. IL: interleuquina; NK: Natural killer; IFN: interferón gamma; FNT: factor de necrosis tumoral; PCR: proteína $C$ reactiva. 


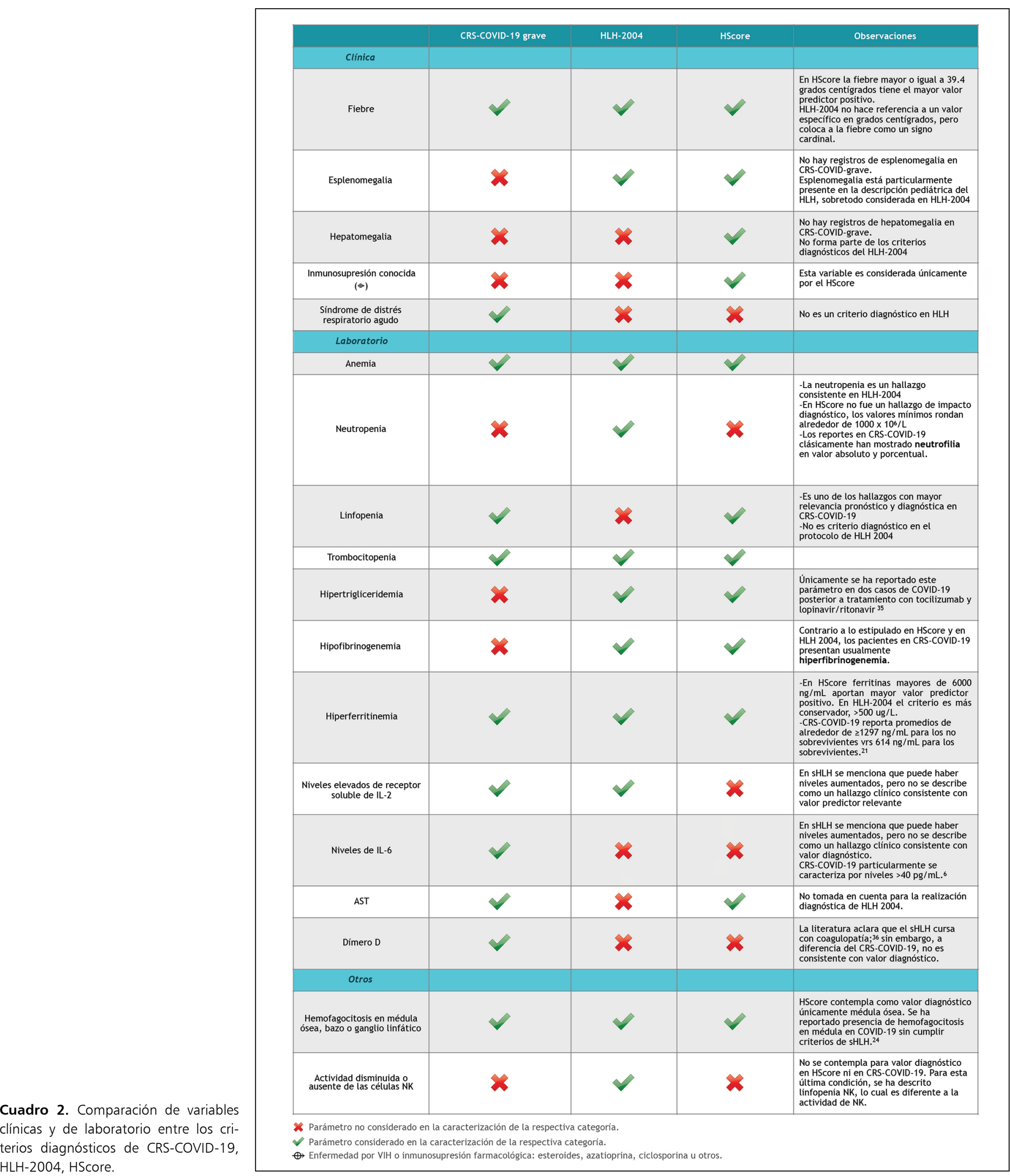


en muerte celular, destrucción de órganos linfáticos como timo y bazo por el propio virus, apoptosis linfocítica por la disregulación citoquínica e inhibición de los linfocitos T por acidosis metabólica hiperlactatémica en el paciente crítico $^{16}$.

Los reportes hasta el momento de CRS-COVID-19 han documentado linfopenia, a expensas de disminución de linfocitos $\mathrm{T} \mathrm{CD}^{+}$, así como de linfocitos $\mathrm{CD}^{+} / \mathrm{CD}^{+}$, $\mathrm{CD}^{+} / \mathrm{CD}^{+}$y células $\mathrm{NK}$; en relación con agotamiento de estas subpoblaciones debido al proceso inflamatorio persistente y consecuente liberación constante de citoquinas por células inmunes, tanto innatas como adaptativas ${ }^{13}$. Un estudio realizado en China, reportó que los pacientes graves poseían un aumento de la relación CD4/CD8, lo cual evidencia importante linfopenia $\mathrm{CD}^{+} / \mathrm{CD}^{+}$en relación con el recuento de los linfocitos $\mathrm{CD}^{+} / \mathrm{CD}^{+}$. Este hallazgo posiblemente sea secundario al agotamiento de linfocitos $\mathrm{T}$ citotóxicos durante su respuesta inmune adaptativa al virus ${ }^{12}$.

Con respecto a la ferritina, característicamente el CRS-COVID-19, al igual que el sHLH, cursa con concentraciones aumentadas de este reactante inflamatorio. La ferritina es una molécula que se encarga de almacenar el hierro en una forma biológicamente disponible para los procesos celulares. La apoferritina es la ferritina libre de hierro; ésta se encuentra compuesta por 24 subunidades. Las mismas pueden ser de dos tipos: la subunidad L y la subunidad $\mathrm{H}^{17}$.

La síntesis de ferritina es mediada predominantemente por citoquinas; sin embargo, también está regulada por estrés oxidativo, hormonas, fenómenos de hipoxia e isquemia, hiperoxemia y replicaciones virales, entre otros, que inducen la síntesis predominantemente de L-ferritina en células hepato-esplénicas ${ }^{17}$.

Por lo tanto, es esperable que en fenómenos inflamatorios intensos exista hiperferritinemia, especialmente cuando hay hipoxemia acompañante, ya que, estas concentraciones aumentadas de ferritina inducen mecanismos inmunosupresores con el motivo de controlar el estado hiperinflamatorio adyacente, a través de anergia de linfocitos $\mathrm{T}$, inhibición de los linfocitos $\mathrm{B}$, supresión de la fagocitosis por granulocitos y regulación de la granulo-monocitopoyesis ${ }^{18}$. Paradójicamente, la ferritina también favorece mecanismos pro-inflamatorios como la síntesis de IL-1 $\beta^{19}$. De tal manera, que dependiendo de los receptores que utilicen las subunidades mencionadas de la ferritina, la molécula podría ejercer, tanto mecanismos pro-inflamatorios como inmunosupresores ${ }^{20}$.

En sHLH, el mayor valor predictor positivo para la ferritina en el puntaje del HScore es un valor $>6.000$ $\mathrm{ng} / \mathrm{mL}$. Valores inferiores a $2.000 \mathrm{ng} / \mathrm{mL}$ no son asignados con puntaje diagnóstico ${ }^{10}$. Por su parte, el valor diagnóstico en el protocolo HLH 2004 es > 500 ng/mL, siendo este mucho más inclusivo con los valores ${ }^{9}$. En el 
CRS-COVID-19, la hiperferritinemia es característica; sin embargo, no alcanza valores tan altos como los pautados en HScore (Cuadro 1) ${ }^{21}$. De manera comparativa, son mucho más similares las concentraciones de ferritina de sHLH por HScore y CRS-COVID-19 que en relación con HLH-2004.

El fibrinógeno es otro de los parámetros de laboratorio que requiere mención, debido a que, en el sHLH, el valor diagnóstico es mayor cuando el paciente cursa con hipofibrinogenemia. Sin embargo, los reportes del CRSCOVID-19 describen que los casos graves se caracterizan por concentraciones aumentadas del fibrinógeno en conjunto con elevaciones del dímero $\mathrm{D}^{15,22}$. Este hallazgo, refleja la presencia de un estado pro-trombótico adyacente, razón por la cual, se ha reportado el uso de heparina de bajo peso molecular en los pacientes con CRS-COVID-19 $\mathrm{y}$ concentraciones aumentadas de dímero $\mathrm{D}^{22}$.

El protocolo HLH-2004 fue elaborado para el diagnóstico y manejo de la HLH primaria del paciente menor de 18 años ${ }^{9}$, lo cual limita la aplicación de sus conceptos a la población adulta; en ese sentido, el HScore vino a abrir un abanico de posibilidades mucho más amplio, permitiendo predecir la utilidad del tratamiento en cualquier edad. Aunado a esto, los criterios de la medición del receptor soluble de IL-2, así como la determinación de la actividad de las células NK, convierte en poco práctico la utilización de los criterios HLH-2004, para el ejercicio clínico rutinario. Basado en estas observaciones, y dada la similitud que encontramos entre los criterios HScore y CRS-COVID-19, es posible que HScore sea una herramienta más sensible en la identificación temprana del paciente con CRS-COVID-19, especialmente en el cuadro grave, que incluso podría traslaparse con HLH. Eventualmente en estos casos, el paciente podría beneficiarse de un tratamiento agresivo del componente inflamatorio. Conforme a lo estipulado en el HScore, el mayor valor predictor para el diagnóstico de sHLH es el resultado de un puntaje mayor a 169 , con una sensibilidad de $93 \%$ y especificidad de $86 \%$ para el diagnóstico ${ }^{10}$.

Con respecto a la hemofagocitosis en COVID-19, el primer reporte de casos clínicos que reveló la presencia de sHLH por COVID-19 fue un reporte de cuatro autopsias, donde se encontró presencia hemofagocitosis en tres casos, distribuidos en los siguientes tejidos: en ganglios linfáticos ( 2 casos) y en bazo ( 1 caso). Curiosamente, ninguno en médula ósea. Solo un caso del total, cumplía con un HScore de 217 puntos, cumpliendo así con el puntaje requerido para el diagnóstico de $\mathrm{sHLH}^{23}$. Estos hallazgos se refuerzan con un estudio francés, que reportó tres casos de COVID-19 con hemofagocitosis en médula ósea; sin embargo, en ningún caso se logró establecer diagnóstico de $\mathrm{sHLH}^{24}$. Estos estudios sustentan que en COVID-19, la presencia de hemofagocitosis no es suficiente para afirmar el diagnóstico de sHLH; en general, esto aplica para cualquier condición, pues como se ha mencionado, deben cumplirse una serie de criterios diagnósticos bien definidos (ya sea por HLH-2004, o HScore 2014), para confirmar este diagnóstico. Por otra parte, un estudio inglés de 40 pacientes con COVID-19 grave, reportó que sólo en tres casos $(7,5 \%)$ se logró documentar un HScore $>169$ puntos, demostrando así, que el sHLH por COVID-19 es infrecuente ${ }^{25}$.

En 2017 fue publicado por Kyriazopoulou y cols., un estudio de cohorte griega, en el cual se describe un grupo de pacientes con sepsis de comportamiento hiperinflamatorio semejante a $\mathrm{HLH}^{26}$. Ante la sospecha clínica, se utilizó el HScore para aproximar el diagnóstico; sin embargo, el puntaje fue modificado ante la no realización de aspirado de médula ósea a los pacientes del estudio. Debido a esta situación, se modificó el HScore original y se retiró el criterio de hemofagocitosis; razón por la cual, se optó denominar a esta condición "síndrome de activación macrofágica-like" "27. Consecuentemente, los autores del estudio disminuyeron el puntaje de mayor sensibilidad para el diagnóstico de HLH (169 puntos) a 151 puntos $^{26}$. Sin embargo, es necesario destacar que esta modificación fue validada únicamente para este estudio clínico, además de que se excluyeron los pacientes con infección por VIH, y esta condición, como se menciona en el Cuadro 2, representa también un puntaje relevante en el HScore original. De manera tal, debe tomarse en cuenta, que utilizar el puntaje de 151 como valor corte para aproximar con mayor sensibilidad y especificidad el diagnóstico de sHLH en el contexto de sepsis, no es una conducta validada fuera del estudio mencionado; no es conforme al HScore original y eventualmente no existe evidencia suficiente para extrapolar este corte del puntaje a CRS-COVID-19.

\section{Conclusiones}

El CRS secundario a COVID-19 grave presenta una gran similitud con el síndrome hemofagocítico secundario por lo que el estudio de las variables clínicas y de laboratorio descritas en esta revisión podrían ser de ayuda en la identificación temprana de casos que se puedan beneficiar de terapia inmunosupresora. Dentro de las herramientas diagnósticas con las que se cuenta hasta el momento para el diagnóstico de sHLH, se concluye que el HScore podría orientar el diagnóstico de una manera más práctica que los criterios diagnósticos de HLH-2004, ya que, esta última herramienta fue realizada con el fin de clasificar al paciente pediátrico con HLH primario y se ha extrapolado al paciente adulto con HLH secundario; mientras que HScore 2014 fue diseñado específicamente para el diagnóstico del sHLH o reactivo y validado en la población adulta. Con las ex- 
son desconocidos hasta el momento; lo cual difiere del sHLH por virus de Epstein-Barr por ejemplo, en el cual, el beneficio ya ha sido consistentemente demostrado. La linfopenia descrita en los casos críticos de COVID-19, posiblemente limite la aplicación clínica de VP-16 en CRS-COVID-1931-32; sin embargo, a dosis bajas no es una intervención que del todo sea descartada ${ }^{33-34}$, aún más en el contexto de que es una terapia mucho menos costosa que los fármacos biológicos. Eventualmente se requieren ensayos clínicos al respecto.

A pesar de que HScore es una herramienta que podría orientar el diagnóstico de sHLH en el contexto del CRS-COVID-19, de una manera más práctica que los criterios clásicos descritos del HLH-2004, su aplicación en COVID-19 se encuentra limitada debido a la ausencia de características claves del estado hiperinflamatorio de COVID-19 que sí destacan en HLH.

Hasta el momento, los reportes de sHLH en COVID-19 con puntaje de HScore $>169$ son escasos.

Agradecimientos: A Rodrigo Ovares Arroyo por su colaboración y apoyo constante.

\section{Referencias bibliográficas}

1.- Lin L, Lu L, Cao W, Li T. Hypothesis for potential pathogenesis of SARS-CoV-2 infection-a review of immune changes in patients with viral pneumonia. Emerg Microbes Infect. 2020; 9 (1): 727-32. doi: 10.1080/22221751.2020.1746199.

2.- Sarzi-Puttini P, Giorgi V, Sirotti S, Marotto D, Ardizzone S, Rizzardini G, et al. COVID-19, cytokines and immunosuppression: what can we learn from severe acute respiratory syndrome? Clin Exp Rheumatol. 2020; 38 (2): 337-42. PMID 32202240.

3.- Ferrara J L, Abhyankar S, Gilliland D G. Cytokine storm of graft-versus-host disease: a critical effector role for interleukin-1. Transplant Proc. 1993; 25 (1 Pt 2): 1216-7. PMID 8442093.

4.- LoVerde D, Iweala O I, Eginli A, Krishnaswamy G. Anaphylaxis. Chest 2018; 153 (2): 528-43. doi: 10.1016/j. chest.2017.07.033.

5.- Lee D W, Gardner R, Porter D L, Louis C U, Ahmed N, Jensen M, et al. Current concepts in the diagnosis and management of cytokine release syndrome. Blood 2014; 124 (2): 188-95. doi: 10.1182/ blood-2014-05-552729.

6.- Nicastri E, Petrosillo N, Ippolito G, D’Offizi G, Marchioni L, Ascoli Bartoli T, et al. National Institute for the Infectious Diseases "L. Spallanzani" IRCCS. Recommendations for COVID-19 Clinical Management. Infect
Dis Rep. 2020; 12 (1). 8543. doi: 10.4081/ idr.2020.8543.

7.- Al-Samkari H, Berliner N. Hemophagocytic lymphohistiocytosis. Annu Rev Pathol Mech Dis. 2018; 13 (1): 27-49. doi: 10.1146/annurevpathol-020117-043625.

8.- Carter S J, Tattersall R S, Ramanan A V. Macrophage activation syndrome in adults: recent advances in pathophysiology, diagnosis and treatment. Rheumatology 2019; 58 (1): 5-17. doi: 10.4081/idr.2020.8543.

9.- Henter J-I, Horne A, Aricó M, Egeler R M, Filipovich A H, Imashuku S, et al. HLH-2004: Diagnostic and therapeutic guidelines for hemophagocytic lymphohistiocytosis. Pediatr Blood Cancer 2007; 48 (2): 124-31. doi. 10.1002/pbc. 21039

10.- Fardet L, Galicier L, Lambotte O, Marzac C, Aumont C, Chahwan D, et al. Development and validation of the HScore, a Score for the diagnosis of reactive hemophagocytic syndrome. Arthritis \& Rheumatol. 2014; 66 (9): 2613-20. doi: 10.1002/art.38690.

11.- Li X, Geng M, Peng Y, Meng L, Lu S Molecular immune pathogenesis and diagnosis of COVID-19. J Pharm Anal 2020; 10 (2): 102-8. S2095177920302045. doi: 10.1016/j. jpha.2020.03.001.

12.- Wang W, Liu X, Wu S, Chen S, Li Y, Nong $\mathrm{L}$, et al. The definition and risks of cytokine release syndrome-like in 11 COVID-19-infected pneumonia critically ill patients: disease characteristics and retrospective analysis. Intensive
Care Med. 2020; 222 (9): 1444-51. doi: 10.1101/2020.02.26.20026989.

13.- Wan S, Yi Q, Fan S, Lv J, Zhang X, Guo $\mathrm{L}$, et al. Characteristics of lymphocyte subsets and cytokines in peripheral blood of 123 hospitalized patients with 2019 novel coronavirus pneumonia (NCP). Hematology; 2020 doi/10.1101/2020.02.10.20021832.

14.- Qin C, Zhou L, Hu Z, Zhang S, Yang S, Tao $\mathrm{Y}$, et al. Dysregulation of immune response in patients with COVID-19 in Wuhan, China. Clin Infect Dis 2020. Jul 28; 71 (15): 762-8. doi: 10.1093/cid/ciaa248.

15.- Wang Y, Yao L, Zhang J-P, Tang P-J, Ye Z-J, Shen $\mathrm{X}-\mathrm{H}$, et al. Clinical characteristics and laboratory indicator analysis of 69 covid-19 pneumonia patients in Suzhou, China. BMC Infect Dis. 2020; 20 (1): 747. doi: 10.2139/ ssrn. 3550041.

16.- Tan L, Wang Q, Zhang D, Ding J, Huang Q, Tang Y-Q, et al. Lymphopenia predicts disease severity of COVID-19: a descriptive and predictive study. Sig Transduct Target Ther. 2020; 5 (1): 33. doi: 10.1038/s41392-020-01484.

17.- Rosário C, Zandman-Goddard G, MeyronHoltz E G, D'Cruz D P, Shoenfeld Y. The hyperferritinemic syndrome: macrophage activation syndrome, Still's disease, septic shock and catastrophic antiphospholipid syndrome. BMC Med. 2013; 11 (1): 185. doi: 10.1186/1741-7015-11-185.

18.- Hann H W, Stahlhut M W, Lee S, London W T, Hann R S. Effects of isoferritins on human 
granulocytes. Cancer 1989; 63 (12): 2492-6. PMID: 2720599.

19.- Ruddell R G, Hoang-Le D, Barwood J M, Rutherford P S, Piva T J, Watters D J, et al. Ferritin functions as a proinflammatory cytokine via iron-independent protein kinase $\mathrm{C}$ zeta/nuclear factor kappaB-regulated signaling in rat hepatic stellate cells. Hepatology 2009; 49 (3): 887-900. doi: 10.1002/hep.22716.

20.- Wigginton J M. Reversal of ferritin-mediated immunosuppression by levamisole: a rationale for its application to management of the acquired immune deficiency syndrome (AIDS). Med Hypotheses 1995; 44 (2): 85-8. doi: 10.1016/0306-9877(95)900756.

21.- Green M S. Did the hesitancy in declaring COVID-19 a pandemic reflect a need to redefine the term? The Lancet. 2020; 395 (10229): 1034. doi: $10.1016 /$ S01406736(20)30630-9.

22.- Tang N, Bai H, Chen X, Gong J, Li D, Sun $\mathrm{Z}$. Anticoagulant treatment is associated with decreased mortality in severe coronavirus disease 2019 patients with coagulopathy. J Thromb Haemost. 2020; 18 (5): 1094-9. doi: $10.1111 /$ jth.14817.

23.- Prilutskiy A, Kritselis M, Shevtsov A, Yambayev I, Vadlamudi C, Zhao Q, et al. SARS-CoV-2 infection associated hemophagocytic lymphohistiocytosis: an autopsy series with clinical and laboratory correlation. Am J Clin Pathol. 2020; 154 (4): 466-74. doi: 10.1101/2020.05.07.20094888.

24.- Debliquis A, Harzallah I, Mootien J Y, Poidevin A, Labro G, Mejri A, et al.
Haemophagocytosis in bone marrow aspirates in patients with COVID-19. Br J Haematol. $2020 \mathrm{Jul} ; 190$ (2): e70-e73 bjh.16860. doi: 10.1111/bjh.16860

25.- Wood H, Jones J, Hui K, Mare T, Pirani T, Galloway J, et al. Secondary HLH is uncommon in severe COVID-19. Br J Haematol. 2020; 190 (5): e283-e285. bjh.16934. doi: 10.1111/bjh.16934.

26.- On behalf of the Hellenic Sepsis Study Group, Kyriazopoulou E, Leventogiannis K, NorrbyTeglund A, Dimopoulos G, Pantazi A, et al. Macrophage activation-like syndrome: an immunological entity associated with rapid progression to death in sepsis. BMC Med. 2017; 15 (1): 172. doi: 10.1186/s12916-0170930-5.

27.- La Rosée P, Horne A, Hines M, von Bahr Greenwood T, Machowicz R, Berliner N, et al. Recommendations for the management of hemophagocytic lymphohistiocytosis in adults. Blood. 2019; 133 (23): 2465-77. doi: 10.1182/ blood.2018894618.

28.- Cao W, Liu X, Bai T, Fan H, Hong K, Song H, et al. High-dose intravenous immunoglobulin as a therapeutic option for deteriorating patients with coronavirus disease 2019. Open Forum Infect Dis. 2020; 7 (3): ofaa102. doi: 10.1093/ ofid/ofaal 102

29.- Keith P, Day M, Perkins L, Moyer L, Hewitt $\mathrm{K}$, Wells A. A novel treatment approach to the novel coronavirus: an argument for the use of therapeutic plasma exchange for fulminant COVID-19. Crit Care. 2020; 24 (1): 128, s13054-020-2836-4. doi: 10.1093/ofid/ofaa102.
30.- La Rosée P. Treatment of hemophagocytic lymphohistiocytosis in adults. Hematology 2015; 2015 (1): 190-6. doi: 10.1182/ asheducation-2015.1.190.

31.- Ray-Coquard I, Borg C, Bachelot T, Sebban C, Philip I, Clapisson G, et al. Baseline and early lymphopenia predict for the risk of febrile neutropenia after chemotherapy. $\mathrm{Br}$ J Cancer 2003; 88 (2): 181-6. doi: 10.1038/ sj.bjc. 6600724 .

32.- Campian J L, Ye X, Brock M, Grossman $\mathrm{S}$ A. Treatment-related lymphopenia in patients with stage iii non-small-cell lung cancer. Cancer Investig. 2013; 31 (3): 183-8. doi: 10.3109/07357907.2013.767342.

33.- Hamizi K, Aouidane S, Belaaloui G. Etoposidebased therapy for severe forms of COVID-19. Med Hypotheses. 2020; 142: 109826. doi: 10.1016/j.mehy.2020.109826.

34.- Takami A. Possible role of low-dose etoposide therapy for hemophagocytic lymphohistiocytosis by COVID-19. Int J Hematol. 2020; 112 (1): 122-4.doi: 10.1007/ s12185-020-02888-9.

35.- Morrison A R, Johnson J M, Ramesh M, Bradley P, Jennings J, Smith Z R. Acute hypertriglyceridemia in patients with COVID-19 receiving tocilizumab. J Med Virol. 21 2020; 92 (10): 1791-2. doi: 10.1002/ jmv.25907.

36.- Valade S, Mariotte E, Azoulay E. Coagulation disorders in hemophagocytic lymphohistiocytosis/macrophage activation syndrome. Crit Care Clin. 2020; 36 (2): 415-26. doi: 10.1016/j.ccc.2019.12.004. 